\title{
Whipping Behavior of Rice Flour Dough
}

\author{
Chiaki ICHIKAWA $^{1}$, Takahiro FUKUNAGA ${ }^{1}$, Daitaro ISHIKAWA ${ }^{2}$, and Tomoyuki FuJII ${ }^{1}$ \\ ${ }^{1}$ Graduate School of Agricultural Science, Tohoku University, 468-1 Aramaki Aza Aoba, Aoba-ku, Sendai, Miyagi 980-8572, Japan \\ 2 Faculty of Agriculture, Fukushima University, 1 Kanayagawa, Fukushima, Fukushima 960-1296, Japan
}

\begin{abstract}
In this study, the bubbles in rice flour dough were investigated under a constant temperature. The bubble size distribution is important for the control of food texture. If bubble sizes depend mainly on the inertial force, viscous force, and surface tension, then the normalized mean bubble diameter should be a function of the Reynolds number and Weber number. We obtained experimental data using a hand mixer, and compared the properties of doughs prepared using six rice flours; each flour was prepared through a different milling process. We also added the size effect of the rice flour particles as the Bond number. Furthermore, we performed a dynamic wettability test to estimate the wettability of the rice flour surface. The results of this test were described well by the Washburn equation, and $d_{c} \cos \theta$ was calculated as a wettability parameter (where, $d_{c}$ : effective diameter of a capillary in a powder bed, $\cos \theta$ : the contact angle). The mean bubble diameter $\left(d_{b m}\right)$ generated by whipping was expected to be affected by the thickness $(d)$ of the rod of the mixer, its movement speed, physical properties of the material, and gravitational acceleration. Therefore, dimensionless mean diameter $\left(d_{b m} / d\right)$ was expressed by a dimensionless equation. The empirical equation obtained was generally applicable to the variety of materials selected for this study.
\end{abstract}

\section{Introduction}

Rice flour has many properties that are advantageous for human health, such as high digestibility, low calories (low absorption potential of oil), and functional components, as compared to wheat flour (Sanchez et al., 2006; Sivaramakrishnan et al., 2004). In particular, the increase in the incidence of celiac disease, which is a digestive issue caused by an immunological reaction to gluten, has increased the demand for gluten-free products in the last two decades (Rai et al., 2018). Rice flour is a key alternative to flour for minimizing food allergies, and many products based on rice flour, such as breads, cakes, and noodles, are commercially available.

However, gluten is an important determinant of the texture of food products, since its network structure holds air and provides elasticity (Okunishi, 2015). The texture of rice flour-based products without gluten is typically moist and chewy Okunishi, 2015). Although these textures of rice flour-based products are unique and appealing, the inflexibility is an obstacle to the expansion of rice flour-based products. Moreover, the processing of cellular foods is usually optimized for flour, so that the favorable texture of rice flour-based food products is not obtained by the simple replacement of flour with rice flour (Torbica et al., 2010). In particular, the optimization of process control methods is essential for the development of new rice flour-based foods with desirable textures (Fitzgerald et al., 2003; Torbica et al., 2010).
Our research group has studied the powder properties of rice flour subjected to different milling techniques to appropriately control the production process. Fujii and Shoji (2012) have reported that the surface and internal structure of rice flour, subjected to milling, forms a coreshell structure. Shoji et al. (2012) have also found that hydration behavior is closely related to the internal structure of rice flour. Moreover, Ishikawa et al. (2017) demonstrated that adsorption properties might be related to the internal structure, and showed the potential of developing optimally controlled production of rice flour based on mechanical shock in the milling process. However, precise analyses of the process control methods for rice flour production are still needed.

Rice flour-based cellular foods have been prepared from rice flour dough with air-liquid-solid phases. As rice flour dough is a foam with many bubbles, investigations of the distribution of bubble sizes during the whipping process are important for the optimization of texture. Therefore, the aim of this study was to evaluate the whipping properties of dough with three phases containing rice flour and soymilk. In this study, the powder properties of rice flour were investigated, and dimensionless parameters were analyzed to construct an appropriate process control system for rice flour-based food products.

\section{Materials and Methods}




\subsection{Materials}

The white rice yielded 2015 year at Miyagi Perfecture, Japan was used for the material of rice flour. Six rice flours, referred to as RFa-RFf, were prepared. RFa was produced by initial milling with a $3.0 \mathrm{~mm}$ screen size and secondary milling at $0.3 \mathrm{~mm}$ under $3,600 \mathrm{rpm}$ using a pin mill device. RFb was pulverized by high-speed milling at $10,000 \mathrm{rpm}$. For RFc, the white rice was milled by pin milling under 3,600 rpm and then pulverized by highspeed milling at $11,000 \mathrm{rpm}$ by the wet grinding method. RFd was produced by pin milling at 3,600 rpm and then by high-speed milling at $10,000 \mathrm{rpm}$. RFe and RFf were pulverized under the same conditions, other than the milling time. The milling time was twice as long for the former than for RFd and four times as long for the latter than for RFd. Rice flours excluding RFc were prepared by the dry grinding method. To investigate the effects of the liquid phase, sucrose was added to unadjusted soymilk containing $12 \%$ soy solids (Kitano Daizu, TaishiSyokuhin) and $10-40 \%$ solutions were prepared.

\subsection{Methods}

\subsubsection{Powder properties}

The particle size distribution was measured using a particle size analyzer (Laser Micron Sizer LMS-2000e; SEISHIN Enterprise Co., Ltd., Tokyo, Japan) by the laser diffraction method. This instrument has a helium neon laser and solid laser as red and blue light sources. Consequently, particle size can be measured at the submicron order.

The particle density of each powder sample was measured by the liquid displacement method using a GayLussac-type Pycnometer (Model 82-2355; Sansyo Co., Ltd., Tokyo, Japan). 2-Propanol was used as the dispersion medium and vacuum-drying was performed under $0.07 \mathrm{MPa}$ for $5 \mathrm{~h}$. Density was measured at $25^{\circ} \mathrm{C}$ and the specific weight of 2-propanol was $0.78084 \mathrm{~g} / \mathrm{cm}^{3}$

The dynamic wetting test of each powder sample was performed using a hydration meter (type: TU-011; Hatsuratsu Co., Sendai, Japan). A sample cup of $40 \mathrm{~mm} \Phi$ and $30 \mathrm{~mm}$ in height was filled with the sample powder and set on a transparent tank. The tank was connected to a water bath. The height of water surface in the bath was reduced by hydration toward the powder bed by capillary pressure. The water was supplied from a reservoir by a micro-pump because the water level was returned. The time course of the volume of water supplied was monitored for $3 \mathrm{~h}$ at intervals of $15 \mathrm{~s}$ The course of the squared hydrate volume was fitted by the Washburn equation (Eq.1), which explains capillary penetration. In this study, hydration of the powder bed was regarded as a capillary phenomenon.

$$
V_{L}^{2}=(S \cdot \varepsilon)^{2} \frac{r \cdot \gamma_{w} \cos \theta}{2 \cdot \eta_{w}} t
$$

where $V_{\mathrm{L}}$ is the hydration volume, $t$ is the hydration time, $S$ is the cross-sectional area of the powder bed, $\varepsilon$ is porosity, $r$ is the capillary radius, $\gamma_{w}$ is the water surface tension, $\eta_{w}$ is the water viscosity, and $\theta$ is the contact angle. In this study, $\gamma_{w}$ and $\eta_{w}$ were set to $72.6 \mathrm{~N} / \mathrm{m}^{2}$ and 1.0026 $\mathrm{mPa} \cdot \mathrm{s}$, respectively.

The slope of the hydration curve obtained by the change in $V_{L}^{2}$ over time was used to obtain the wetting parameter, $C \cos \theta$, as follows:

$$
\operatorname{Cos} \theta=(\text { Slope }) \cdot \frac{\eta_{w}}{\gamma_{w}}
$$

Simultaneously,

$$
C=(S \cdot \epsilon) \cdot \frac{r}{2}
$$

Therefore, when the capillary radius $(r)$ and porosity $(\varepsilon)$ are constant during the hydration period, the wettability parameter $d_{c} \cos \theta$ can be quantitatively defined. In this study, the capillary diameter $(2 r)$ was defined as $d_{c}$.

\subsubsection{Physical properties of the liquid phase}

Sucrose in the concentration range of $0-40 \%$ was added to soymilk and agitated using a magnetic stirrer. The surface tension of the solution was measured using a surface tension meter (SURFACE TENSIOMETER LBVP-A3; KYOWA KAIMENKAGAKU Co., Ltd., Tokyo, Japan). The density was measured following the methods described in section 2.2.1.

\subsubsection{Whipping properties of rice flour-based dough prepared with soymilk}

Soymilk with sucrose was subjected to mixing using a hand mixer (Tescom THM26) at $1250 \mathrm{rpm}$ for $1 \mathrm{~min}$ and then $50 \mathrm{~g}$ of each rice flour was added to the solution. Each solution was subjected to mixing using the same whipper at $1250 \mathrm{rpm}$ for $10 \mathrm{~min}$, and rice flour-based dough with soymilk was prepared.

The bubbles in the rice flour dough were investigated under a constant temperature. A slide glass was dropped on the dough and settled on a microscope. Images of 1740 $\times 1180$ pixels were obtained and transferred to Gel-Pro Analyzer using a microscope equipped with a CCD camera. Bubbles were identified in each image, and the diameter of bubbles was measured. A total of 512 bubbles were measured in each image and the mean bubble size was calculated.

\section{Results and Discussion}

\subsection{Evaluation of powder properties by dimensionless parameters}


Particle size measurements for rice flours are shown in Table 1. The volume-based mean particle sizes ranged from 52.73 to $241.9 \mu \mathrm{m}$. We calculated number-based mean particle sizes from volume-based mean particle sizes for each sample. Number-based mean particle size was $1.650-7.218 \mu \mathrm{m}$. In this study, we used the numberbased mean particle size as the mean particle size $\left(d_{p}\right)$ of each sample.

Table 1. Summary of volume-based and number-based mean particle sizes for each rice flour

\begin{tabular}{cccccc}
\hline & $\mathbf{D}[\mathbf{4 , 3}]$ & $\mathbf{d}(\mathbf{0 . 1})$ & $\mathbf{d}(\mathbf{0 . 5})$ & $\mathbf{d}(\mathbf{0 . 9})$ & $\boldsymbol{d}_{\boldsymbol{p}}$ \\
\hline $\mathrm{RFa}$ & 185.2 & 49.83 & 176.4 & 325.1 & 6.076 \\
$\mathrm{RFb}$ & 241.9 & 43.97 & 165.6 & 493.8 & 7.218 \\
$\mathrm{RFc}$ & 198.0 & 34.36 & 152.4 & 376.4 & 5.631 \\
$\mathrm{RFd}$ & 52.73 & 9.632 & 44.25 & 108.2 & 1.650 \\
$\mathrm{RFe}$ & 144.0 & 19.46 & 86.90 & 225.6 & 2.158 \\
$\mathrm{RFf}$ & 226.1 & 31.55 & 134.3 & 480.7 & 5.442 \\
\hline
\end{tabular}

$\mathrm{D}$ [4.3]: volume-based mean particle size ; $\mathrm{d}(0.1)$ : maximum particle diameter is less than $10 \%$ of the sample falls ; $d(0.5)$ : maximum particle diameter below which $50 \%$ of the sample falls ; $d(0.9)$ : maximum particle diameter below which $90 \%$ of the sample falls ; $d_{p}$ : number-based mean particle size calculated from $\mathrm{D}[4,3]$.

The surface state of a particle is estimated well by the Bond number. To investigate the surface state of rice flour, the Bond number $(\mathrm{Bo})$ based on a particle was calculated by the ratio of the contribution of gravity to the surface tension as follows:

$$
B o=\frac{\rho_{p} d_{p}^{2} g}{\gamma_{L}}
$$

where $B o$ is the Bond number based on a particle, $\rho_{p}$ is the particle density, $d_{p}$ is the particle diameter, $g$ is gravity, and $\gamma_{L}$ is the liquid surface tension.

The Bond number calculated using the density of particles, surface tension, and gravity was in the range of $6.2 \times 10^{-7}$ to $1.2 \times 10^{-5}$.

The course of the squared hydrate volume is summarized in Figure 1. A linear increase in the squared volume of liquid was observed. The trend was well described by the Washburn equation (see Eq. 1). The wettability parameter $d_{c} \cos \theta$ was calculated using the slope of the regression in Figure 1.

It was assumed that wettability parameter $d_{c} \cos \theta$ is affected by the particle size; thus, a dimensionless wettability parameter was obtained by the particle size $\left(d_{p}\right)$. The dimensionless parameter of wettability $d_{c} \cos \theta / d_{p}$ ranged from 2.561 to 4.260 among rice flours.

A two-dimensional plot of the Bond number and $d_{c} \cos \theta / d_{p}$ is shown in Figure 2. The points were clearly distributed in the two-dimensional map of Bond number and $d_{c} \cos \theta / d_{p}$. Thus, these results suggested that rice flour subjected to different milling procedures could be classified by their Bond number and $d_{c} \cos \theta / d_{p}$.

\subsection{Physical properties of the liquid phase}

The surface tension of the sample solution-based soymilk was $0.0451-0.0466 \mathrm{~N} / \mathrm{m}$ for $0-40 \%$ sucrose. The density of these solutions ranged from 1.115 to $1.404 \mathrm{~kg} / \mathrm{m}^{3}$ for $0-40 \%$ sucrose.

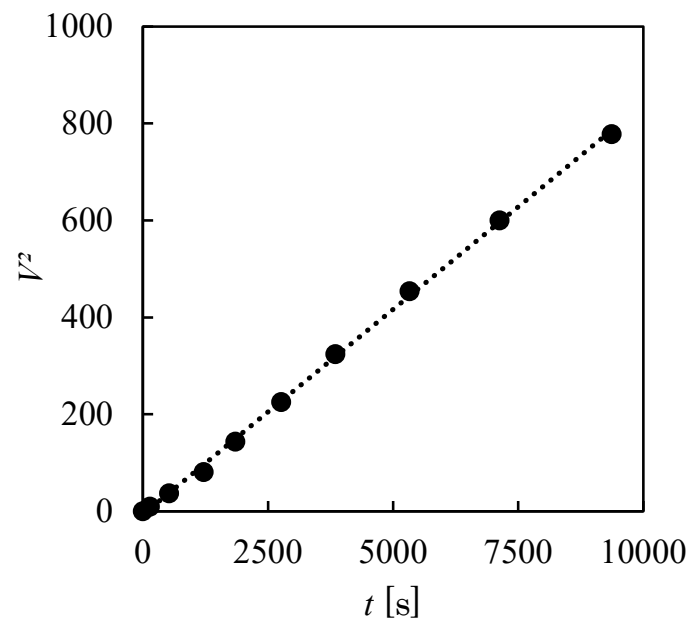

Figure 1. Course of the squared volume of hydration over time.

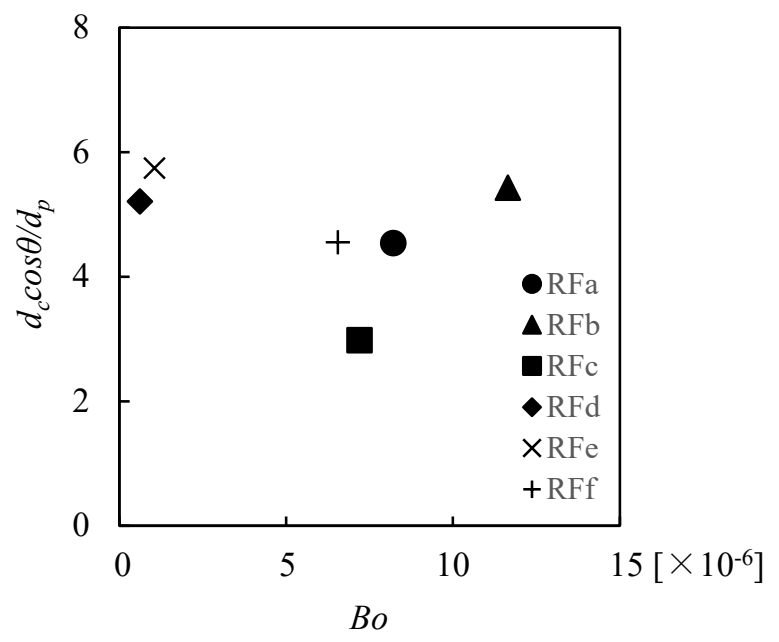

Figure 2. Plot of the Bond number and dimensionless parameter for wettablilty $d_{c} \cos \theta / d_{p}$

\subsection{Whipping behavior}

Bubble size was assumed to depend mainly on three kinds of force, i.e., inertial force, viscous force, and surface tension, in this study. Thus, the normalized mean bubble diameter should be described by a function of the Reynolds number $(R e)$ and Weber number $(W e)$. Re and $W e$ were defined as follows:

$$
\begin{gathered}
R e=\frac{u d \rho_{L}}{\eta_{L}} \\
W e=\frac{u^{2} d \rho_{L}}{\gamma_{L}}
\end{gathered}
$$


where $u$ is the whipping speed, $\rho_{L}$ is the liquid density, $d$ is the whipper size, $\eta_{L}$ is the liquid viscosity, and $\gamma_{L}$ is the liquid surface tension.

$B o$ and $W e$ accounted for surface tension such that the whipping behavior was dependent on the interfacial effect of the solid and liquid phases. Thus, the estimation index $d_{b m} / d$ including the effect of solid and liquid phases was constructed as follows to understand the whipping behavior based on the coefficient $A$ and the power values $k, l, m$, and $n$.

$$
\frac{d_{b m}}{d}=A(W e)^{k}(R e)^{l}(B o)^{m}\left(\frac{d_{c} \cos \theta}{d_{p}}\right)^{n}
$$

The relationship between the normalized mean bubble size and estimation index is shown in Figure 3. A strong linear correlation was observed. Thus, this analysis successfully demonstrated that bubble size could be estimated by dimensionless parameters. Moreover, the coefficient $A$ and the power values $k, l, m$, and $n$ were $0.0370,-0.379,0.228,0.0495$, and 0.289 , respectively. Consequently, the simple quadratic equation might clarify the contribution of each parameter. In particular, the contributions of $R e$ and $d_{c} \cos \theta / d_{p}$ to mean bubble size were high and $W e$ had a negative effect on bubble size.

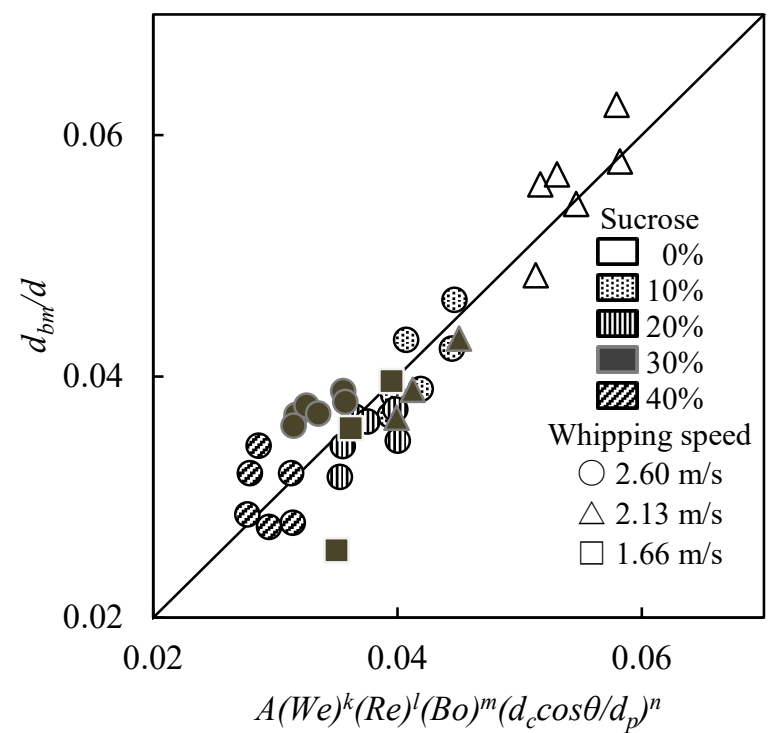

Figure 3. Relationship between $d_{b m} / d$ and the estimated dimensionless parameter.

The quest for the optimization of process control methods is essential for the development of new rice flour-based cellular foods in response to food allergies. The aim of this study was to investigate the whipping behavior of rice flour dough. Six types of rice flour were prepared by different milling processes. To evaluate the powder properties of rice flour, the particle density, surface tension, and wettability were investigated. A two- dimensional plot of the relationship between Bond number and the wettability parameter $d_{c} \cos \theta / d_{p}$ clearly suggested that differences in the powder properties of rice flour could be evaluated by particle size and wettability. The form of foam strongly depended on inertial, viscous, and interfacial effects, and the normalized mean bubble diameter, reflecting the whipping behavior, was predicted by the Reynolds number, Weber number, and Bond number. The dimensionless mean diameter $\left(d_{b m} / d\right)$ was expressed by a dimensionless equation. These results successfully demonstrated the potential to evaluate the whipping process by dimensionless parameters.

\section{Acknowledgements}

The rice flours used in this study were provided by Hatsuratsu Inc. This study was financially supported by the Iijima Memorial Foundation for the Promotion of Food Science and Technology, 2019.

\section{References}

Fitzgerald, M. A., M. Martin, R. M. Ward, W. D. Park, and H. J. Shead; "Viscosity of Rice Flour: A Rheological and Biological Study," J. Agric. Food Chem., 51(8) 2295 -2299 (2003)

Fujii, T. and M. Shoji; "The Fine Structure of Rice-starch," (in Japanese) Jpn. J. Appl. Glycosci., 2, 92-96 (2012)

Ishikawa, D., I. Sawa, Y. Sekiyama, A. Horigane, T. Okunishi, K. Fujii, and T. Fujii; "Study on the Change in Powder Properties of Rice Flour by Different Milling Processes," J. Appl. Glycosci., 64, 109-114 (2017)

Okunishi, T.; "Future on Rice Flour Bread," (in Japanese) Jpn. J. Cook Sci., 48(6), 385-391 (2015)

Rai, S., A. Kaur, and C. S. Chopra; "Gluten Free Products for Celiac Susceptible People," Front. Nutr., 5(116) https://doi.org/10.3389/fnut.2018.00116 (2018)

Sanchez, H. D., C. A. Osella, and M. A. de la Torre; "Optimization of Gluten-Free Bread Prepared from Corn Starch, Rice Flour, and Cassava Starch," J. Food Sci., 67(1), 416-419 (2006)

Shoji, N., Y. Hanyu, S. Mori, S. Hatakenaka, M. Ikeda, C. Togashi, and T. Fujii; "Evaluation of Powder and Hydration Properties of Rice Flour Milled by Different techniques," (in Japanese) Jpn. J. Food Eng., 59, 192-198 (2012)

Sivaramakrishnan, H. P., B. Senge, and P. K. Chattopadhyay; "Rheological Properties of Rice Dough for Making Rice Bread," J. Food Eng., 62, 37-45 (2004)

Torbica, A., M. Hadnađev, and T. Dapčević; "Rheological, Textural and Sensory Properties of Glutenfree Bread Formulations Based on Rice and Buckwheat Flour," Food Hydrocoloids, 24, 626-632 (2010) 